\title{
¿Energía incluyente? Expectativas de participación en el Chile de hoy
}

\author{
Inclusive energy? Expectations towards Participation in Contemporary Chile
}

Marco Billi

Escuela de Gobierno, Universidad Adolfo Ibáñez, Chile

RESUMEN

Desde el punto de vista sociopolítico, la distinción de igualdad/ desigualdad se relaciona estrechamente con la de inclusión/ exclusión: así, las desigualdades inherentes en los problemas socioambientales pueden ser vistas, a la vez, como problemas de inclusión: inclusión en el espacio de la decisión, inclusión en el espacio de los afectados, inclusión en el espacio de los compensados. La multiplicación de movimientos de protesta dirigidos a visibilizar estas desigualdades e incluir a los afectados en el debate político manifiesta justamente esta relación. Sin embargo, al duplicarse la distinción el resultado se complejiza: inclusión/ exclusión e igualdad/ desigualdad son dos esquemas de observación transversales el uno al otro, que al cruzarse multiplican las posibilidades. A partir de esta herramienta teórica, apoyada en desarrollos de la Teoría de Sistemas, y de la escuela francesa de las Nuevas Desigualdades, la ponencia observará las actuales tendencias hacia la participación en las políticas energéticas chilenas, y en particular en los Estándares de Participación desarrollados por el programa ministerial Energía 2050, reflexionando sobre las expectativas de igualdad e inclusión a las que ellos tratan dar respuesta, y sobre los límites que estas mismas expectativas conllevan.

PALABRAS ClaVE: Energía 2050; Políticas participativas; Inclusión; Desigualdad; Conflictos socioambientales

ABSTRACT

From the socio-political point of view, the distinction equality/ inequality appears firmly related to the one of inclusion/ exclusion. Thus, the inequalities embedded in socio-environmental problems may also be seen as problems of inclusion: inclusion in the space of decisions, inclusion in the space of those affected by those decisions, inclusion in the space of those receiving compensations for them. This connection is made manifest by the multiplication of movements aimed at making these inequalities visible and including the affected in the political debate. However, by being duplicated, the distinction is also made more complex: inclusion/ exclusion and equality/ inequality are two observation schemes orthogonal to each other, so that crossing them multiplies the possible results. From this perspective, based on developments of the Theory of Social Systems, and the french school of New Inequalities, the paper will look at the current trends towards participation within Chilean energy policy, and particularly to the Estándares de Participación (Standards of Participation) built into the governmental program Energía 2050. Specifically, it will offer a reflection on the expectations to equality and inclusion these programs try to respond to, and on the limits that these same expectations imply.

KEYWORDS: Energía 2050; Participative politics; Inclusion; Inequality; Socio-environmental conflicts

\footnotetext{
REVISTA MAD - REVISTA DEL MAGÍsTER EN ANÁLISIS SISTÉMICO APLICADO A LA SOCIEDAD ISSN 0718-0527

Facultad de Ciencias Sociales, Departamento de Antropología. Universidad de Chile www.revistamad.uchile.cl
}

DOI: http://dx.doi.org/10.5354/0718-0527.2016.40618 


\section{INTRODUCCIÓN ${ }^{1}$}

Como toda observación social, también la afirmación de que la relación entre sociedad y medioambiente es (o no es) un problema de desigualdad, está sujeta a los esquemas de observación (también sociales), a través de los cuales se mira esta relación. Diversos lentes configuran una diferente relación y con ella, diferentes problemas. Así, desde la jerga económica se hablaría quizás de desigualdad en la atribución de recursos naturales, desde el sistema jurídico de una desigualdad de derechos, y desde la política de una desigualdad de poder, etc.

Desde esta última mirada, se observaría al medioambiente en términos de la función de la política, esto es, producir decisiones colectivamente vinculantes y por lo tanto la desigualdad se referiría, por un lado, al poder de tomar estas mismas decisiones, y secundariamente, a las consecuencias de estas decisiones. Pero con ello esta desigualdad vendría a tomar también la forma de una inclusión: inclusión en el espacio de toma de decisiones e inclusión en el espacio de consecuencias. Inclusión que es socialmente determinada en ambos casos y que posee consecuencias sociales.

En la sociedad moderna estos dos espacios parecen quedar fuertemente relacionados: a medida que se reconoce que un cierto grupo es afectado por una decisión, se tiende a darle poder de influir sobre ella, o donde no es posible de compensarlo por las consecuencias de una decisión de la que no fue responsable. Esta es la esencia finalmente de la democracia, de la participación ${ }^{2}$. Así actúan los movimientos de protesta ambientales: haciendo escuchar su voz como afectados para poder ganar el poder de decisión, o bien compensación (Fitoussi \& Rosanvallon 2010; Luhmann 2007). De este modo, se mueven en dirección opuesta a los procesos participativos con los que el Estado busca expandir la inclusión en sus decisiones.

\footnotetext{
${ }^{1}$ Versión revisada y corregida de la ponencia presentada en la mesa "Los problemas socioambientales como problemas de desigualdad" presentada en el seminario "Sociedad y medio ambiente: un problema de desigualdad" organizada por el Magister en Análisis Sistémico aplicado a la Sociedad (MaSS). Santiago de Chile, 22/10/2015.

${ }^{2}$ Remitir la democracia a la determinación individual, es un tópico, por lo menos, de toda teoría de justicia democrática de tipo liberal (desde John Rawls a todos sus críticos y seguidores): esto a su vez implica cierta resistencia a las medidas que impactan sobre esta libre determinación (afectando a individuos independientemente de sus decisiones) de no ser que pueda justificarse por causas superiores de interés colectivo. Queda de todos modos por decidirse cuáles de estas causas pueden considerarse justificadas; al respecto, ver los debates presentados en Barry (2001) y Galston (1995).
} 
Ahora bien, si inclusión e igualdad están conectadas, esto no significa que la una conlleve necesariamente a la otra. Son dos distinciones que se cruzan, creando espacios transversales.

En el presente trabajo se reflexiona sobre la intersección entre inclusión/ exclusión e igualdad/ desigualdad en el marco de los procesos participativos de la sociedad moderna, tomando como caso significativo a los procesos participativos que en Chile se están actualmente llevando a cabo en el campo de la política energética, ya que, como se argumentará, representan un ejemplo paradigmático de los problemas inherentes a este tipo de fenómeno.

Antes de ello, sin embargo, se hace necesaria una digresión teórica, dirigida a resumir la manera en que éstas dos distinciones se han conectado en la sociología contemporánea; para ello, es de ayuda considerar las diferentes perspectivas que apuntan a la observación del individuo en la sociedad.

\section{INDIVIDUO Y SOCIEDAD EN LA SOCIOLOGÍA: LOS CONCEPTOS DE IGUALDAD E INCLUSIÓN}

La relación entre la sociedad y el individuo es un problema clásico de la sociología, y ocupa un rol importante en el trabajo de diferentes autores: el individuo, que antaño era el único portador de comportamiento provisto de sentido (Weber 1993), queda encerrado en la "jaula de la obediencia" por el imperativo de racionalidad moderno (Weber 1964), y la ambigüedad del sistema normativo en Émile Durkheim (1990). Posteriormente, tras haber quedado completamente anulado frente a los poderes económicos en Max Horkheimer y Theodor Adorno (1997), para luego ser integrado en los roles institucionalizados determinados por los sistemas sociales y sus medios simbólicos en Talcott Parsons (1965; 1981), acaba por ser derechamente separado de la sociedad en Niklas Luhmann (1992; 1998; 2007). De acuerdo con éste, la sociedad se compone sólo de comunicaciones, las cuales se reproducen autopoiéticamente a sí mismas; el individuo, pese a ser necesario para la comunicación, se distingue netamente de ella y queda por lo tanto colocado en el entorno de la sociedad. Sin embargo, desde esta nueva posición, el individuo, en tanto sistema psíquico a su vez autónomo y autopoiético recupera un espacio para su propia individualidad, que se vuelve a la vez independiente de las determinaciones estructurales de la sociedad y presupuesto para el propio 
funcionamiento de los sistemas sociales y el aumento constante de su complejidad.

Esta complejidad, a la vez, termina por fragmentar la capacidad del Yo de remitirse a las expectativas normativizadas institucionalmente del Otro generalizado, y con ello el individuo se encuentra obligado a escribir su propia biografía a través de elecciones siempre contingentes en un mundo que sin embargo no puede controlar (Robles 2005). De forma parecida, la modernización reflexiva de Ulrich Beck (1992) implica que los individuos, liberados de las determinaciones estructurales de un mundo globalizado en que cada decisión se vuelve riesgosa (la "Sociedad del Riesgo"), pero también privados de las certezas de la sociedad industrial, deben hacer frente a la compulsión a la búsqueda de nuevas certezas (en un proceso de constante e inacaba individualización).

En este contexto, los conceptos de inclusión y exclusión juegan un rol importante, enmarcando la relación individuo-sociedad en una forma de dos lados (Spencer-Brown 1979) donde sin embargo sólo el lado de la inclusión queda marcado comunicativamente, mientras que la exclusión queda definida justamente por la ausencia de toda indicación que permita dar cuenta comunicativamente de la relevancia social de los individuos (Luhmann 1995). La sociedad, de esta forma, incluye (o no incluye) a los individuos en tanto "personas", "asignándoles lugares en cuyo marco pueden actuar de acuerdo a expectativas complementarias" (Luhmann 2007: 492). Pero ni inclusión ni exclusión quedan definidas como categorías absolutas: nadie puede estar plenamente incluido o plenamente excluido, sino que se multiplican las posibilidades diferenciadas de inclusión (exclusión) dentro de distintos espacios funcionales, con autonomía el uno del otro: así, ser rico no implica necesariamente tener salud, ni amor, ni poder (político), sino que cada sistema reclama para sí la operación de selección de las referencias en base a una racionalidad propia que no puede ser determinada por los otros sistemas.

Desde el punto de vista del individuo, la inclusión se configura en primer lugar en términos de opciones disponibles para participar en los rendimientos sociales; lo que a su vez influye sobre las posibilidades que el individuo tiene a disposición para configurar su propia biografía a partir de la autoconfrontación consigo mismo dentro de las opciones individualizadas que la inclusión permite. Al reverso, la exclusión se traduce en un diario problema de "recomposición de la propia biografía cotidiana [cuando en el medio de] las inseguridades manufacturadas a las que están expuestos los que viven en la periferia globalizada [de una so- 
ciedad del riesgo] la autoconfrontación del sujeto consigo mismo es desregulada y no asistida (por nadie), pero al mismo tiempo la búsqueda del otro es obligada y no escogida" (Robles 2005: 11-12). En otras palabras, el 'haz de tu vida lo que te parezca' de la individualización incluida se contrapone al 'arréglatela como puedas' de la individuación excluida.

Desde el punto de vista de la sociedad, esta situación se configura en términos de la capacidad de los diferentes sistemas sociales de ofrecer alternativas para cubrir las diferentes opciones de participación que las personas desde su individualidad pueden escoger (Mascareño \& Carvajal 2015). Y es en este sentido que esta distinción ha ido tomando un rol creciente dentro de la sociedad moderna, particularmente en el análisis y práctica de políticas públicas, sobre todo gracias a su capacidad de indicar personas que "están dentro o fuera de criterios específicos de consideración social, por sobre o por debajo de ciertos límites que materializan la diferencia" (Mascareño \& Carvajal 2015: 2), y con ello, de servir como criterio capaz de guiar las decisiones estratégicas-políticas de intervención social. Sin embargo, en su versión tradicional y binaria, este esquema es demasiado rígido y limitado, generando demarcaciones estáticas adosadas a personas, incapaces de dar cuenta de la complejidad de la sociedad moderna. Esto ha sido relevado por una variedad de autores, desde Georg Simmel a Durkheim y Parsons, pasando por el ya citado Luhmann, que han buscado de varias formas complejizar y enriquecer la distinción. Pero es sólo con la ruptura del esquema binario que empieza a hacerse posible explicar eficazmente las paradojas de la sociedad moderna.

Esta ruptura, a su vez, puede tomar varias formas: una de ellas puede verse en la distinción entre rendimientos sistémicos y redes sociales. Para Fernando Robles esto significa duplicar la distinción en primaria y secundaria, indicando en la segunda la posibilidad de "acceder a redes interaccionales (intra y extrafamiliares) de influencia y de favores de las que se obtengan provecho, desde la red de apoyo poblacional al joint venture entre política y criminalidad, hasta la ayuda del hurto y el asalto para acceder al consumo" (Robles 2005: 22). De esta manera, a las situaciones arquetípicas de inclusión o exclusión de ambas dimensiones, primaria/sistémica y secundaria/redes, se suman posibles paradigmas de inclusión excluida (inclusión en las prestaciones sistémicas, pero no en las redes sociales) o exclusión incluida (exclusión desde las prestaciones de los diferentes sistemas, pero inclusión a las redes sociales de apoyo). 
Una opción alternativa, y potencialmente complementaria, se refiere a cruzar el esquema inclusión/ exclusión con otra distinción, ortogonal a la primera, de igualdad/ desigualad.

Estos últimos conceptos, que gozan de una posición central dentro de la sociología tradicional, tienen en la Teoría de Sistemas Sociales un papel ambivalente, debido en gran parte a la posición central que esta asigna a la desigualdad en sociedades con diferenciación de centroperiferia o estratificada, siendo en ambos casos una distinción primaria alrededor de la cual estos tipos de sociedad se estructuraban (paralela a la de sistema/entorno), y que dentro de ellas se reproducía. Al contrario, en la moderna sociedad funcionalmente diferenciada, cada sistema es encargado de una función específica, desigual respecto a la de los otros, pero de acceso igualitario; y es más, aun siendo desiguales en sus funciones, los sistemas parciales de esta sociedad son iguales en términos de rango, no pudiendo establecerse una jerarquía entre ellos: en otras palabras, estos son "iguales respecto de su desigualdad" (Luhmann 2007: 613). En este sentido, como sugiere Armin Nassehi la estratificación parecería permanecer como un "parasito de la diferenciación funcional" (Nassehi 1999: 128, citado en Cadenas 2012: 6), que se reproduce aprovechando los rendimientos sistémicos pese a no tener funcionalidad.

No obstante, en la sociedad funcionalmente diferenciada, el principio de acceso igualitario a las prestaciones sistémicas no impide que, a nivel de sus rendimientos efectivos (inclusión/ exclusión) puedan aparecer desigualdades, las que ya no se alinean, como ocurría con las formas previas de diferenciación, con las situaciones de exclusión, sino que "tanto en el lado de la igualdad como en el de la desigualdad existen inclusiones y exclusiones, por tanto no se presenta una identificación entre ningún lado de ambas formas" (Cadenas 2012: 15).

A partir de aquí, diferentes caminos son posibles para comprender el rol que la desigualdad sigue jugando en la sociedad contemporánea: uno de ello es reintroducir en la misma distinción de igualdad y desigualdad aquella ya observada en Robles (2005) entre rendimientos sistémicos y redes sociales, y de esta forma buscar aquellas conformaciones de desigualdad que resultan más incluyentes o más excluyentes ${ }^{3}$.

\footnotetext{
${ }^{3}$ Este es el camino que sigue Hugo Cadenas, condensando la distinción sistema/redes sociales a través de los medios simbólicamente generalizados, e identificando a las segundas como parte de un nuevo medio simbólico de la "influencia": a partir de ahí, el autor diferencia entre una desigualdad incluyente, fundada en la convertibilidad basal entre medios simbólicos, que deja abiertas y generalizadas las posiciones ventajosas y desventajosas dentro de los sistemas, y una desigualdad excluyente, fundada en la
} 
Otra opción es volver al individuo, y considerar los diferentes efectos que diferentes posibles constelaciones de inclusión y exclusión signifiquen para él, y para las diferentes opciones que estas les ofrezcan (o limiten) en la construcción de su propia biografía.

\section{NUEVAS DESIGUALDADES Y CONSTELACIONES DE INCLUSIÓN/ EXCLUSIÓN}

Esta última perspectiva es la que adoptan Jean-Paul Fitoussi y Pierre Rosanvallon (2010): si bien reconocen que el cambio social ha hecho desaparecer formas de inclusión estables como el grupo, la clase o la comunidad, ellos no remiten este hecho a la desaparición de modalidades de estratificación y jerarquización típicas de formas de diferenciación social previas, sino a una creciente individualización de dichas modalidades. Como consecuencia de ello, las referencias al estatus se vuelven inestables, obligando a pasar de la descripción de identidades colectivas y estructuras de exclusión a una visión más procesual e individualizada de la misma.

Coherente con ello, al lado de una dimensión estática y estructural de la desigualdad - fundada en diferencias de ingresos, patrimonio, educación, etc.-, aparece otra dinámica e individualizada, implícita en la trayectoria de vida de cada persona ${ }^{4}$. Esto, a su vez, repercute sobre la forma con las que los individuos experimentan sus relaciones, que se fragmentan de acuerdo con los cambios que los individuos viven en sus trayectorias de inclusión y exclusión. Así:

“i) quien antes era "un igual" (misma profesión, mismo espacio laboral, mismos lugares de residencia), ahora es distinto: 'Un ejecutivo desempleado desde hace tiempo, por ejemplo, no vive su situación únicamente en términos de baja de los ingresos: se siente en primer lugar excluido del mundo de los ejecutivos' [Fitoussi y Rosanvallon, 2010, pág. 74], y ii) quien antes era distinto, ahora es un 'igual desigual': 'Entre un pequeño empresario en quiebra, un ejecutivo desocupado y un asalariado con un empleo precario, con el paso del tiempo las diferencias de ingreso terminan por borrarse, sin que pese a eso aquellos formen una categoría homogénea' [Fitoussi y Rosanvallon, 2010, pág. 76]" (Mascareño \& Carvajal 2015: 6)

Ahora bien, si se cruza esta desigualdad dinámica con el esquema de inclusión /exclusión se obtienen dos resultados. Por un lado:

convertibilidad secundaria mediada justamente por la influencia, a la cual la efectividad de los medios de comunicación simbólicamente generalizados queda sometida (Cadenas 2016: 19).

${ }^{4}$ Algo parecido se ofrece en Luhmann con el concepto de "carrera" (Esposito, Corsi \& Baraldi 1996: 87). 
Las posibilidades descriptivas se multiplican y las paradojas reales se visibilizan: los que se hallan incluidos pueden estar desigualmente incluidos. Con ello se pueden observar distintos grados de exclusión en la inclusión, como sucede en un sistema educativo cuya calidad es altamente dependiente de las disponibilidades monetarias de las familias [Repetto, 2011]. O también: quienes están excluidos pueden ser objeto de inclusión igualitaria por la vía de desigualdades institucionalmente aceptadas que excluyen a otros, como en el caso de las cuotas de participación femenina en política [Ríos, 2008]. (Mascareño \& Carvajal 2015: 6-7)

Por otro lado, esto permite introducir un estándar normativo para la evaluación de diferentes inclusiones y exclusiones, es decir, se puede estar excluido/a de forma más o menos igual y por lo tanto más o menos legítimamente en términos sociales. Como ya notaba Niklas Luhmann (2010), la exclusión es un proceso fundamental de toda organización y con ello de una gran variedad de procesos sociales, pero no por esto se verá a ésta siempre como injusta, desigual o socialmente indeseable. Pocos protestarán si una empresa decide no incluir (no contratar) a todas las personas que se presenten frente a sus puertas (quizás con la excepción de los directos afectados de esta decisión), pero si se descubre que esta exclusión deriva de criterios ajenos al funcionamiento normal del sistema - carrera, currículo, prestaciones, sueldos (Luhmann 2007; Luhmann \& De Giorgi 1992: 246ss.), incluyendo variables por ejemplo de género, o pertenencia religiosa. Incluso, para suplir a esta exclusión, pueden introducirse dinámicas de inclusión (a su vez desigual) vistas como compensatorias -como cuotas de género, para personas incapacitadas, etc. (Mascareño \& Carvajal 2015).

A partir de estos antecedentes, Aldo Mascareño y Fabiola Carvajal proponen considerar conjuntamente, la individualización de los procesos de inclusión/ exclusión, y en particular "la capacidad de selección de las personas en relación con las opciones que ellas consideren más apropiadas para la realización de sus planes de vida" (Mascareño \& Carvajal 2015: 7); por el otro, "la capacidad de distintos niveles de organización social de ofrecer tanto un número suficiente de alternativas, como una adecuada diversidad de ellas, de modo tal que la opciones de las personas sean cubiertas (en profundidad y amplitud) por la disponibilidad institucional" (Mascareño \& Carvajal 2015: 8).

Esta estrategia permite construir constelaciones de inclusión/ exclusión que visibilizan la interpenetración entre ambas dimensiones, 
dando forma a las múltiples combinaciones de inclusión en la exclusión y exclusión en la inclusión que pueden observarse en la sociedad moderna.

\section{EXCLUSIONES, DESIGUALDADES Y MEDIO AMBIENTE: LA INCLUSIÓN POR} RIESGO Y LA COMPENSACIÓN

Entre las diferentes constelaciones que los autores identifican, dos parecen particularmente interesantes para analizar las desigualdades relacionadas con el medio ambiente: la primera se relaciona con un nivel organizacional y por lo tanto con las decisiones tomadas respecto a variables medioambientales.

En este caso, debe considerarse que toda decisión incorpora siempre un elevado nivel de contingencia en la selección entre diferentes alternativas, la cual sólo puede hacerse desde la información disponible al momento de tomar la decisión, sin que sea posible predecir (aunque sí, reducir a un cálculo) sus consecuencias futuras.

Desde el espacio interno de la decisión, esta indeterminabilidad se observa como "riesgo" y su cálculo se considera necesario para poder decidir, y conseguir cualesquiera sean las metas que la decisión se propone: sin riesgo no hay decisión ni acción (Luhmann 1992).

Sin embargo, desde el espacio exterior de la decisión, desde quienes están excluidos de ella, esta indeterminabilidad se observa como "peligro" de verse afectados por efectos (impredecibles) de decisiones riesgosas en las que no se ha tomado parte. Quedan, por así decir, en una inclusión excluyente.

Esta situación se radicaliza aún más en la Sociedad del Riesgo moderna, en la que estas situaciones riesgosas se hacen a la vez más globales e incontrolables, y más conectadas con las decisiones individuales sobre la recomposición de las biografías cotidianas. De todos modos: "trátese de riesgos (observados como producto de una decisión) o de peligros (observados como externos) en ninguno de estos casos puede contarse con una distribución equitativa de ventajas y desventajas y en ambos casos la magnitud de riesgos y peligros depende de la observación que de ellos se haga" (Robles 2005: 11).

Esto conduce por lo menos a dos niveles de problemas: por un lado, constituye una fuente constante de desigualdad; y por el otro, hace dependiente esta misma desigualdad (o por lo menos, la forma con la que esta se observa comunicacionalmente, lo que desde el punto de vista de la sociedad es lo mismo) de la posibilidad los individuos de responsabilizarse 
respecto a estos riesgos, es decir, de tomar parte en las decisiones en los que ellos se consideran como riesgo -y no como peligro (Mascareño \& Carvajal 2015: 9).

En ambos sentidos, esto constituye un serio problema para la democracia, por lo que proliferan respuestas dirigidas a expandir el espacio de inclusión decisional -inclusión por riesgo- a través de mecanismos más o menos eficaces de participación en el proceso decisional (ya sea a través de representación, consulta pública, etc.). El problema de estos procesos es que, por definición, nunca van a poder alcanzar a todos los potenciales afectados.

Frente a esto, aparece una segunda respuesta de carácter institucional, dirigida a reequilibrar el efecto de estas mismas decisiones sobre los individuos, particularmente quienes han sido finalmente excluidos de la decisión, y por lo tanto en situación de peligro: se trata de la compensación.

Al opuesto de la exclusión por peligro, la inclusión compensatoria es una exclusión incluyente (Mascareño \& Carvajal 2015: 10): se reconocen a ciertas personas como excluidas de forma desigual y se las incluye en el espacio de la compensación. Sin embargo, esta modalidad no da respuesta a todo lo que la exclusión de la decisión comporta y, de hecho, crea nuevos problemas de desigualdad.

En primer lugar, la compensación no puede prevenir el peligro y de hecho es consecuencia de éste: es justamente al hacer notoria la vulnerabilidad y la desigualdad producida, por ejemplo, por catástrofes ambientales o por los efectos de procesos económicos o políticos sobre el territorio, que se reconoce el derecho a una compensación. Pero el daño, por así llamarlo, queda hecho y se registra en la memoria del sistema: la compensación sólo puede sustituir lo que se perdió, y ésta siempre podrá observarse como más o menos adecuada (y así ser objeto de crítica).

Además, si la compensación, que por definición es pensada como transitoria, se ofrece frente a situaciones de carácter permanente o de largo plazo, ella genera una condición de dependencia en los compensados. Así, por ejemplo, frente a una situación de catástrofe ambiental, como el huracán Katrina de New Orleans del 2005 (Iversen \& Armstrong 2008), podría parecer que en lo inmediato la compensación logra reducir la desigualdad -en sus síntomas-, sin embargo, si no alcanza a reestablecer un rango de selectividad suficiente entre las personas, es decir, si no logra dirigirse a las raíces de la desigualdad, poniendo a las personas en condición de acceder a los rendimientos sociales de forma 
independiente de la compensación misma (reestablecer la producción económica, la infraestructura de salud, el sistema educativo), se genera un círculo vicioso que hace permanente a la necesidad de compensación y, a la vez, institucionaliza la desigualdad. De la misma forma, las compensaciones ofrecidas en virtud de los daños ambientales generados por alguna actividad económica, como la minería o grandes proyectos termo- o hidroeléctricos, serán eficaces remedios a la desigualdad si logran reestablecer las condiciones de participación social previas a la intervención; si, por el contrario, se dan en términos de beneficios económicos o parecidos para compensar para la pérdida de rentabilidad de la agricultura, la pesca u otros trabajos, generarán una desigualdad institucionalizada y una dependencia de las compensaciones.

Finalmente, la compensación misma depende de una decisión que reconozca a excluidos desiguales en situación de necesidad de compensación y, al reconocerlos, los incluye. Sin embargo, esta decisión será marcadamente contingente y, por lo tanto, siempre puede causar otras exclusiones y, con ellas, críticas. Así, los que no han sido reconocidos como afectados por una catástrofe o una intervención territorial, siempre podrán ver la compensación que los supuestos afectados reciban como exclusión y como fuente de desigualdad injustificada. Más aún, si como resultado -no previsto- de las compensaciones, los no compensados se ven mermados en el acceso a alguna prestación local, por efecto de una priorización de los supuestos afectados. En este caso, la compensación misma genera riesgo - de dependencia- para los compensados, y peligro de tratamiento desigual- para los no compensados.

A continuación, se presenta el caso central para el presente análisis, vale decir, la planificación de proyectos energéticos y sus impactos socioambientales y sociopolíticos.

\section{POLÍTICAS ENERGÉTICAS, CONFLICTOS Y PARTICIPACIÓN}

Los proyectos energéticos se han ido volviendo un tema cada vez más central dentro de la política chilena e internacional, tanto a la necesidad creciente de energía para provisionar el consumo doméstico y el desarrollo económico e industrial, como por las incrementadas presiones hacia la adopción de una matriz energética más sustentable para el país; una que sea idónea para cumplir con las cada vez más estrictas reglamentaciones internacionales y las apremiantes demandas de la ciudadanía y del tercer sector, en términos de mitigación del cambio climático, 
reducción de la contaminación atmosférica y acuífera, eficiencia, disminución de desperdicios etc. A la vez, los últimos años han mostrado la gran complejidad de este ámbito, debido a la gran variedad de actores involucrados, a las diversas racionalidades (técnica, económica, espiritual, política, etc.) que justifican sus demandas y decisiones, y a las expectativas generadas. Así, llaman la atención las dificultades que tienen que superar los proyectos energéticos, muchas veces antes de siquiera comenzar. Adoptando incluso tecnologías renovables, tratando involucrar a los afectados en el proceso de planificación, y cumpliendo al pie de la letra los requerimientos legales, muchos de ellos encuentran fuertes oposiciones por parte de la opinión pública y de la sociedad civil. Estas resistencias conllevan a veces a su inviabilidad, a menudo a la desconfianza (de las poblaciones y comunidades), a retrasos (en los proyectos y compromisos), a incertidumbre y pérdidas económicas (de las empresas), casi siempre a problemas de gobernanza (para el Estado y sus instituciones) y en general a un progresivo aumento de la tensión nacional e internacional para una planificación más inclusiva, participativa, prospectiva y justa (Astorga 2013; Fuenzalida \& Quiroz 2012; Ortiz \& Bourlon 2011; Romero Toledo 2014).

Como posible respuesta, se van multiplicando los programas y los esfuerzos del gobierno y de las propias empresas para generar mecanismos, normas y herramientas que tengan en cuenta estos valores. Entre estos, el programa participativo Energía 2050 del Ministerio de Energía representa un caso particularmente interesante tanto por su ambición, como por su recepción pública.

\section{ENERGÍA 2050 Y LOS ESTÁNDARES DE PARTICIPACIÓN}

Energía 2050 se denomina a un proceso de planificación participativa, derivado de la llamada Política Energética de Largo Plazo, iniciativa inédita en Chile y liderada por el Ministerio de Energía de acuerdo con los compromisos de la Agenda de Energía lanzada por el gobierno de la Presidenta Michelle Bachelet en mayo de 2014. De acuerdo con la Hoja de Ruta del programa: "A través de diversas instancias de discusión y participación, Energía 2050 busca construir una visión compartida para el desarrollo futuro del sector energía con la validación social, política y técnica requerida para transformarse en la política energética que el país necesita" (Ministerio de Energía 2015a: 7). 
En la práctica, esto se traduce en una estrategia energética con hitos y metas concretas para el año 2035 y una visión a más largo plazo, llegando al año 2050. Dicha estrategia fue construida a partir de un proceso de 18 meses de duración, iniciado en agosto de 2014 y concluido en abril de 2016, en el cual se contemplaron diversas instancias de discusión y participación, incluyendo un Comité Consultivo de carácter estratégico, compuesto por actores clave del sector; una serie de grupos de trabajo temáticos de carácter técnico (eficiencia energética, energía termoe hidroeléctrica, ERNC, redes de transmisión, innovación energética, asuntos indígenas, etc.); talleres regionales $\mathrm{y}$, siguiendo las recomendaciones de la OECD en cuanto a la validación social de las políticas públicas, una plataforma ciudadana virtual y presencial, donde se dispone la información relevante del proceso -sus objetivos, formas de participar, plazos, insumos técnicos, preguntas frecuentes-, se reciben comentarios por parte de la ciudadanía y se informa del material disponible, de modo de alcanzar una participación ciudadana amplia.

Como parte de este proceso, el programa ha contemplado el desarrollo de una Guía de Estándares de Participación para el desarrollo de proyectos de energía, orientada a establecer las formas de diseñar y ejecutar proyectos energéticos en estrecha relación con las comunidades y con la participación de las mismas en las decisiones que las afectan (Ministerio de Energía 2015b). En este sentido, la finalidad última de estos estándares es "Promover que los proyectos de inversión energética que requiere el país, se desarrollen en base al ejercicio y respeto de los derechos sociales, ambientales, territoriales, culturales (tangibles e intangibles) $\mathrm{y}$, económicos de personas y comunidades y, que aporten al desarrollo de los territorios relacionados con el proyecto" (Ministerio de Energía 2015b: 6).

Respecto de lo que nos interesa analizar, resulta interesante que estos Estándares, no hagan referencia a un proceso de participación en una decisión de intervención, como puede ser la realización de un proyecto energético específico, ni representan criterios programáticos para la futura toma de decisiones sobre intervenciones de este tipo (como sí ocurre con otros documentos de Energía 2050, como la Hoja de Ruta, la Agenda de Energía, la Estrategia de Largo Plazo, etc.), sino que invocan más bien una participación en las decisiones referidas a la propia participación; es decir, la decisión sobre quiénes -y cómo- estarán incluidos en futuras decisiones. 
$\mathrm{Si}$, como demandarían los ideales democráticos y el discurso instalado en la opinión pública y en los movimientos de protesta socioambientales, se considera que una efectiva participación en las decisiones sea un elemento clave para la promoción de la igualdad, entonces el problema fundamental es cómo hacer frente a la necesaria contingencia de todo proceso participativo, ya que la inclusión de ciertos grupos en un proceso decisional conlleva necesariamente una exclusión de todos los demás. En este sentido, estos Estándares de participación parecerían representar un importante mecanismo técnico-político de reducción de la complejidad dirigido a:

Establecer un marco claro de interacción entre las empresas de energía, las comunidades y el Estado, que reduzca las asimetrías existentes, respete los derechos de las partes, identifique el tipo y grado de participación que se requiere en el desarrollo de los proyectos de energía, y promueva la construcción de acuerdos sostenibles entre los distintos actores y comunidades que comparten un territorio. (Ministerio de Energía 2015b: 6).

Para poder hacerse cargo de dicha tarea, los estándares buscan ser, a la vez, tan precisos como sea necesario para poder actuar como premisa de futuras decisiones, y tan generalizables como para poder aplicarse a una gran variedad de decisiones y potenciales afectados. Para ello, trabajan en 4 niveles, considerando principios de carácter valórico, los que son luego traducidos en criterios tangibles, es decir en dimensiones y variables a considerar, y posteriormente en acciones de implementación, a mantenerse por todo el ciclo de vida del proyecto, y finalmente indicadores para evaluar el proceso y los resultados de la implementación de los Estándares.

El elevado nivel de abstracción de estos Estándares los hace altamente susceptibles a sus propios puntos ciegos, con efectos difícilmente predecibles tanto sobre sus participantes como sus no participantes.

\section{PARTICIPACIÓN, INCLUSIÓN E IGUALDAD}

Partiendo de estas premisas, resulta interesante explorar cuáles pueden ser las expectativas que un programa de este tipo incorpora respecto de la sociedad y qué condiciones pretende poner a la misma, a fin de responder a las diferentes demandas relacionadas con el desarrollo y evaluación de proyectos energéticos. En suma, ¿Qué tipo de forma de inclusión y parti- 
cipación se propone, y cuáles rendimientos y límites pueden esperarse de estos procesos deliberativos respecto a la desigualdad socioambiental?

Para responder a esta pregunta, se propone seguir el esquema de trabajo presentado previamente, mirando los procesos de inclusión/ exclusión y de igualdad/ desigualdad implícitos en el proceso de construcción de los Estándares de participación, tomando en consideración tres puntos de vista: el de aquellos incluidos en el proceso decisional, de los excluidos de dicho proceso y de los compensados por dicha exclusión.

Respecto de la primera dimensión, un primer abordaje puede obtenerse atendiendo a la función de los Estándares de Participación dentro de Energía 2050. En estos Estándares se observa un primer nivel de riesgo de dicho programa que llamaremos técnico, relativo a las consecuencias de las decisiones actuales en términos de matriz energética sobre las futuras condiciones del País. Sobre este tema, se ha destacado que

En un escenario sin cambios (BAU, Business-as-usual), Chile se convertiría en el más grande contaminador per cápita en América Latina y se ubicaría por encima de la mayoría de los países europeos, lo que afectaría gravemente su competitividad a nivel mundial. Esto genera un aumento en su perfil de riesgo y el incumplimiento con su compromiso internacional del $20 \%$ de reducciones de gases de efecto invernadero para el año 2020. Críticamente, Chile generaría el 47\% de su energía a partir de carbón importado para el año 2030, una situación aún más riesgosa que las importaciones de gas natural desde Argentina en la década de 1990 con sus conocidas consecuencias. (Carvalho et al. 2014: 2).

A la vez, toda decisión dirigida a cambiar esta matriz energética conllevará riesgos relacionados con las futuras, y nunca completamente predecibles, consecuencias de estas decisiones, tanto sobre las variables que se quieren afectar como sobre otras no tomadas en cuenta al momento de la decisión. En este primer nivel, los Estándares de participación tomarían la función de mejorar la información disponible para la decisión y, con ello, reducir el riesgo asociado a la misma.

Esta primera observación puede profundizarse observando el documento preliminar de los Estándares de Participación, donde se señala un ulterior y potencial riesgo implícito en su elaboración, relacionado con que:

Los actores se comprometerán a dialogar sin pre-condiciones de obtener un resultado determinado (aunque pueden defender dicho interés con legitimidad en el diálogo), debiendo manejar apropiadamente sus expectativas y moderar 
potenciales acciones especulativas, pues va a existir permanentemente la posibilidad que el proyecto se realice o no se realice. (Ministerio de Energía 2015b: 4).

Esto adquiere una forma más concreta si se considera que, de acuerdo con el documento antes citado:

Un proceso realizado utilizando este estándar, podrá ser calificado de exitoso si la decisión tomada de realizar o no un proyecto energético y las definiciones de cómo realizarlo, han considerado los intereses y necesidades de los distintos actores durante el proceso de diálogo. (Ministerio de Energía 2015b: 4).

En otras palabras, se reconocen por lo menos dos niveles de riesgo: el primero, que está relacionado con los decisores, es que la discusión no llegue a ningún puerto y represente un gasto (de tiempo, energía, recursos) sin resultados. Este es un riesgo común a todo proceso de inversión y, en línea con la teoría económica, puede ser fácilmente valorado con base en la probabilidad percibida que el proceso efectivamente conlleve resultados; lo que, en otras palabras, se transforma en un problema de confianza en el proceso público, el gobierno y el compromiso de todas las partes.

El segundo nivel es más difícil de tratar, porque refiere al riesgo que las decisiones tomadas en relación con los Estándares (y, en general, con el programa Energía 2050) generen consecuencias en relación con procesos decisionales futuros, relacionados con el diseño e implementación de proyectos específicos. Es, por así decirlo, un riesgo de que la decisión actual sea causa de riesgos futuros; el problema, aquí es que no existe ninguna garantía de que estos procesos futuros efectivamente se lleven a cabo, cuándo, bajo qué condiciones y, lo que más importa, a quién tomarán en cuenta. Esto porque la inclusión en decisiones futuras de actores no incluidos en la presente, aporta elementos de indeterminabilidad que finalmente hacen incalculable el riesgo asociado a la Estrategia Energética.

En este sentido, depurando el discurso sobre los Estándares de su componente valórica y utópica, su función -organizacional- podría verse en reducir la contingencia de las futuras decisiones de inclusión y exclusión de actores desde los procesos decisionales y, de este modo, reducir el riesgo asociado. En otras palabras, los Estándares actúan como "premisas de decisión" (Luhmann 2010) para las futuras decisiones, que las hacen por lo menos- calculables en el presente. 
Desde el punto de vista de los que no participan del proceso deliberativo, todo esto toma la forma de una exclusión por peligro, donde el peligro principal es no estar incluidos en la decisión y así estar expuestos al potencial peligro ulterior de estar sujetos a las consecuencias de esas futuras decisiones, ya sea en términos de sus impactos sobre el territorio ( $\mathrm{y}$, consecuentemente, sobre el contexto medioambiental y social) o de las soluciones para las necesidades energéticas de los excluidos, en términos de acceso a la energía, costo, continuidad, etc., las cuales han sido incorporadas dentro del mismo proceso bajo el nombre de "pobreza energética" (Ministerio de Energía 2015a: 11).

Aquí aparecen nuevamente múltiples niveles en lo que refiere a consecuencias y peligros asociados, los que al parecer harían preferible para todos los actores interesados tomar parte del proceso de construcción de los Estándares. De esta forma, podríamos decir que el proceso deliberativo se asegura a sí mismo frente a la necesaria limitación de la inclusión, tal como ocurre con las elecciones democráticas, puesto que es un proceso abierto a todos los interesados y que se preocupa de proporcionar las herramientas mínimas para la participación (información, descentralización, accesibilidad etc.), lo que significa que puede declararse no responsable de eventuales exclusiones ${ }^{5}$. Esto claramente no libera al proceso participativo de las potenciales acusaciones de insuficiente transparencia o accesibilidad -que, por lo demás, nunca podrían evitarse por completo -, pero sí hace menos legítimas y fundadas estas acusaciones, probabilizando la participación por sobre la autoexclusión del proceso.

Más compleja es la situación si se toma en cuenta otro tipo de riesgo/ peligro: no el quedar excluidos de los futuros procesos decisionales, sino estar incluidos, pero con mecanismos no favorables desiguales. Esto es, por ejemplo, lo que acusan los actores de comunidades locales y especialmente la población indígena, al destacar que en el proceso de fijar -a su decir, unilateralmente- las condiciones iniciales del debate, no se reflejarían los reales intereses de los afectados.

De cierta forma, esto es esperable, ya que todo proceso comunicativo -como lo es también el proceso deliberativo- necesita comenzar con premisas, las que a su vez incluyen o excluyen, si no

\footnotetext{
${ }^{5}$ Más precisamente, siguiendo la metodología presentada por Cadenas (2012) podría procesar eventuales exclusiones dentro de la dinámica propia del mismo sistema, justificándolas en términos de falta de relevancia o legitimidad política; en este sentido, toda desigualdad resultante de la exclusión no sería una disfunción, sino un resultado basal y necesario del funcionamiento del propio sistema político.
} 
directamente personas, por lo menos posibilidades comunicativas que estas personas podrían preferir. En el caso de los Estándares de participación, esto se hace más evidente si se considera la explícita fundamentación de éstos sobre principios de carácter valórico, lo que en una sociedad moderna nunca es un camino seguro hacia el consenso, por el contrario, siempre podrán existir comunicaciones que invoquen otros valores, incluso opuestos, y siempre inconmensurables (Luhmann 2007: 320). En relación con las comunidades indígenas, por ejemplo, los principios de Planificación Conjunta y de Desarrollo Local, propuestos por el documento (Ministerio de Energía 2015b), resultan evidentemente incompatibles con las ideas de autodeterminación y de apego a la tradición que algunas de estas poblaciones persiguen (Mariman 2012).

Frente a esto, el programa ha propuesto una Guía de Estándar más específica, dirigida precisamente a los proyectos susceptibles de afectar a pueblos originarios y elaborada especialmente con la participación de los mismos. Esta podría verse como una forma de compensación frente a la desigualdad introducida por el proceso deliberativo: compensación que, sin embargo, ya en sus orígenes no tiene carácter transitorio, sino que es permanente, ya que no refiere sólo al proceso de construcción de los Estándares, sino que derivará en un documento y proceso de participación completamente separado. Esto no hará más que radicalizar las condiciones de desigualdad que, como toda compensación, este proceso genera: desigualdad implícita en la diferencia de trato que reciben las comunidades indígenas y que, los excluidos -los no indígena- podrían ver como un privilegio injustificado, mientras que, para los mismos indígenas, resultará en una legitimación de su condición de diferentes, de excluidos: una inclusión excluyente.

Irónicamente, el mismo programa Energía 2050 proponía entre sus puntos fuertes que, gracias a la participación, "se instala un nuevo enfoque para la relación entre comunidades y el desarrollo energético, transitando desde la lógica actual de compensaciones hacia una que intenta insertar las iniciativas energéticas en el concepto de desarrollo local que buscan y promueven las propias comunidades" (Ministerio de Energía 2015a: 10).

\section{CONCLUSIONES}

En general, estos ejemplos muestran las necesarias limitaciones que tiene que enfrentar un proceso deliberativo dirigido a promover la participación 
y prevenir las desigualdades relacionadas con procesos de intervención territorial y planificación energética, y los impactos ambientales a ellos conectados. Con todo, se trata de un proceso muy ambicioso y valorable en su intención de dar respuesta a las demandas sociales y políticas de inclusión decisional y de mejorar los procesos de toma de decisiones, haciendo más calculables sus riesgos y menos inevitables sus peligros, los cuales, en última instancia, pueden transformarse en riesgos mediante la participación.

Desde el punto de vista de los afectados, esto significa poder contar con nuevas posibilidades institucionalizadas de inclusión, que les restituyen un cierto grado control sobre las inseguridades que cruzan sus vidas y sus elecciones cotidianas, sustentando al reconocerlas sus construcciones de individualidad (Robles 2012) ${ }^{6}$.

Pero los límites señalados no dejan de ser importantes, en la medida en que pueden llevar a observaciones críticas, a pérdidas de confianza, a decisiones poco fundadas o demasiado rígidas, a desigualdades no previstas, etc., como ampliamente observan movimientos de protesta y representantes de comunidades originarias.

Pero estas son todavía observaciones de primer orden.

En el segundo orden parecería urgente repensar, no tanto las características de estas iniciativas, sino la forma de observarlas y las expectativas que fundamentan su evaluación y diseño, lo que a la vez significa preguntarse: ¿Qué nivel de complejidad -del entorno- se proponen abordar y qué nivel de complejidad -programática- es necesaria para ello? (Matus 2012a; Matus 2012b).

Sin eso, se corre el riesgo de caer en una adicción a la compensación en respuesta a exclusiones no previstas, o a inclusiones no eficaces, con el doble resultado de producir inflaciones incontrolables en la complejidad que el sistema político se encuentra obligado a manejar, y también de expandir sus límites externos hasta el punto de poner en crisis la diferenciación interna de otros sistemas que lo rodean, particularmente del sistema económico (Teubner 2011).

Finalmente, a modo de conclusión, se propone una pregunta, con la esperanza de que pueda inspirar las futuras observaciones de políticas participativas, tanto en el campo energético como en otras áreas. ¿Qué pretensiones puede tener de sí misma la participación política? ¿Tal vez

\footnotetext{
${ }^{6}$ Esto de hecho es aún más cierto por lo que refiere a las comunidades originarias, para las cuales el reconocimiento de Estándares separados se transforma en un reconocimiento de su propia diversidad, y con ello, de su identidad individualizada como pueblo (Taylor 2009).
} 
una utópica y normativa inclusión total y perfectamente igualitaria en las decisiones y en sus consecuencias, aún al costo de ignorar la paradoja de que no puede haber inclusión sin exclusión, o igualdad sin desigualdad? O bien, ¿Una inagotable y autorreferente flexibilización de los procesos de inclusión/ exclusión en las decisiones, en consideración de las nuevas y dinámicas trayectorias de desigualdad individualizada características de la sociedad moderna? RM

\section{REFERENCIAS}

Astorga, E. (2013). Conflicto Ambiental en Patagonia. Observaciones sobre la comunicación medioambiental en la región de Aysén a raíz del proyecto Hidroaysen (Tesis presentada para obtener el grado de Magíster en Análisis Sistémico Aplicado a la Sociedad). Santiago de Chile: Universidad de Chile.

Barry, B. (2001). The Strategy or Privatization. En Culture \& Equality. An Egaliatarian Critique of Multiculturalism (Cap. 2, pp. 19-62). Cambridge: Polity Press.

Beck, U. (1992). Risk Society: Towards a New Modernity. London: Sage.

Cadenas, H. (2012). La desigualdad de la sociedad. Diferenciación y desigualdad en la sociedad moderna. Persona y Sociedad, 26(2), 51-77.

Cadenas, H. (2016). Desigualdad social y teoría de sistemas: la importancia de los medios. Economía y Política, 3(1), 41-69.

Carvalho, J., Hidalgo-González, P., Kammen, D., Maxwell, A., Sims, D., \& Finat, C. (2014). Imaginando un Chile Sustentable. Cinco Hallazgos sobre el futuro del sistema eléctrico y energético chileno. Acera \& Universidad de Berkeley. URL: http://www.energia2050.cl/material/461 Consultado en octubre de 2014.

Durkheim, E. (1990). Der Selbstmord. Frankfurt aM: Suhrkamp.

Esposito, E., Corsi, G., y Baraldi, C. (1996). Glosario sobre la teoría Social de Niklas Lukann. México DF: Universidad Iberoamericana.

Galston, W. A. (1995). Two Concepts of Liberalism. Ethics, 105(3), 516-534.

Ministerio de Energía (2015a). Hoja de Ruta 2050. Hacia una energía sustentable e inclusiva para Chile. Santiago, Chile: Comité Consultivo de Energía 2050, Ministerio de Energía, Gobierno de Chile.

Ministerio de Energía (2015b). Guía de Esándar de Participación para proyectos de Energía. Versión preliminar para consulta pública. Santiago, Chile: Energía 2050, División de Participación y Diálogo Social, Ministerio de Energía, Gobierno de Chile.

Fitoussi, J.-P., \& Rosanvallon, P. (2010). La nueva era de las desigualdades. Buenos Aires: Ediciones Manantial.

Fuenzalida, M., \& Quiroz, R. (2012). La dimensión espacial de los conflictos ambientales en Chile. Polis (Santiago), 11(31), 157-168.

Horkheimer, M., \& Adorno, T. (1997). Dialéctica de la Ilustración. Madrid: Trotta.

Iversen, R., \& Armstrong, A. (2008). Hurricane Katrina and New Orleans: What Might a Sociological Embeddedness Perspective Offer Disaster Research and Planning? Analyses of Social Issues and Public Policy, 8(1), 183-209. 
Luhmann, N. (1992). Sociología del Riesgo. Guadalajara, México: Universidad Iberoamericana \& Universidad de Guadalajara.

Luhmann, N. (1995). Inklusion und Exklusion. En Soziologische Aufklärung 6: Die Soziologie und der Mensch (pp. 237-264). Opladen: Westdeutscher Verlag.

Luhmann, N. (1998). Complejidad y modernidad. De la unidad a la diferencia. Madrid: Trotta.

Luhmann, N. (2007). La sociedad de la sociedad. México DF: Universidad Iberoamericana.

Luhmann, N. (2009). La política como sistema. México DF: Universidad Iberoamericana. Luhmann, N. (2010). Organización y Decisión. Mexico DF: Universidad Iberoamericana. Luhmann, N., \& De Giorgi, R. (1992). Teoria della società. Milano: FrancoAngeli.

MAPS Chile. (2014). Opciones de Mitigación para el Cambio Climático. Fase 2: síntesis de resultados. Santiago de Chile: Ministerio del Medio Ambiente.

Mariman, J. (2012). Autodeterminación: Ideas políticas mapuche en el albor del siglo XXI. Santiago de Chile: Lom.

Mascareño, A., \& Carvajal, F. (2015). Los distintos rostros de la inclusión y la exclusión. Revista CEPAL, 116, 131-146.

Matus, T. (2012a). La gestión de la intervención social. Desarrollo de un Modelo de Gestión de Calidad para Programas sociales. FONDEF 2007/2008. Santiago, Chile: Editorial Librosdementira Ltda.

Matus, T. (2012b). Observar la complejidad: Un desafío a las políticas públicas. En H. Cadenas, A. Mascareño, \& A. Urquiza (Eds.), Niklas Luhmann y el legado universalista de su teoría (pp. 205-218). Santiago de Chile: RIL Editores.

Nassehi, A. (1999). Inklusion, Exklusion - Integration, Desintegration. Die Theorie funktionaler Differenzierung und die Desintegrationsthese. En Differenzierungsfolgen. Beiträge zur Soziologie der Moderne (pp. 105-131). Wiesbaden \& Opladen: Westdeutscher Verlag.

Ortiz, P. S., \& Bourlon, F. (2011). Represas en Aysén: ¿Traba o trampolín para el desarrollo turístico regional? Sociedad Hoy, 20, 145-157.

Parsons, T. (1965). El sistema social. Madrid: Revista de Occidente.

Parsons, T. (1981). El aspecto político de la estructura y el proceso sociales. En D. Easton, Enfoques sobre teoría política. Buenos Aires: Amorrortu.

Robles, F. (2005). Contramodernidad y Desigualdad Social: Individualización e individuación, inclusión/exclusión y construcción de identidad. La necesidad de una sociología de la exclusión. Revista Mad, 12, 27-58.

Romero Toledo, H. (2014). Ecología política y represas: Elementos para el análisis del Proyecto HidroAysén en la Patagonia chilena. Revista de geografía Norte Grande, 57, 161-175.

Sen, A. (2009). The Idea of Justice. London: Allen Lane, Penguin Group.

Spencer-Brown, G. (1979). Laws of Form. New York: E. P. Dutton.

Taylor, C. (2009). El multiculturalismo y la política del reconocimiento. México DF: FCE.

Teubner, G. (2011). A Constitutional Moment? The Logics of "Hitting the Bottom". En P. Kjaer, G. Teubner, \& A. Febbrajo (Eds.), The Financial Crisis in Constituional Perspective (pp. 3-42). Oxford: Hart.

Weber, M. (1964). Economía y sociedad. Esbozo de sociología comprensiva. México DF: FCE. 
Weber, M. (1993): Ensayos sobre metodología sociológica. Buenos Aires: Amorrortu.

\section{SOBRE EL AUTOR}

Marco Billi posee el grado de bachiller en Economía y Gestión de las Empresas, con especialización en Mercados e Instituciones Financieras por la Universitá Commerciale Luigi Bocconi de Milán, Italia. Además, posee el grado de Magister en Análisis Sistémico aplicado a la Sociedad por la Universidad de Chile. Es actualmente estudiante del Doctorado en Procesos e Instituciones Políticas de la Escuela de Gobierno de la Universidad Adolfo Ibáñez, Chile. Ha colaborado como investigador para el centro de estudios y agencia formativa acreditada Instituto Mille e una Méta, de Livorno, Italia, como Director de Estudios para el Observatorio Contra el Acoso Callejero Chile, y es actualmente Consultor de Estudios en Inclusión Financiera para la Fundación TechnoServe Chile.

\section{CONTACTO}

Escuela de Gobierno

Universidad Adolfo Ibáñez

Diagonal Las Torres 2640

Santiago, Chile

CP: 7941169

marco.dg.billi@gmail.com

Recibido: Diciembre 2015

Aceptado: Marzo 2016 